\title{
Clinical manifestations, risk factors, and prognostic factors of cytomegalovirus enteritis
}

\author{
Pai-Jui Yeh ${ }^{1}$, Cheng-Tang Chiu ${ }^{2,3}$, Ming-Wei Lai ${ }^{1,3,4}$, Ren-Chin Wu ${ }^{5}$, Chien-Ming Chen ${ }^{6}$, Chia-Jung Kuo ${ }^{2,3}$, \\ Jun-Te Hsu' ${ }^{7}$, Ming-Yao Su ${ }^{3,8}$, Wei-Pin Lin ${ }^{2,3}$, Tsung-Hsing Chen ${ }^{2,3}$ and Puo-Hsien Le 2,3,4*
}

\begin{abstract}
Background: To comprehensively analyze the risk factors, clinical characteristics, outcomes, and prognostic factors of Cytomegalovirus (CMV) enteritis.

Methods: This retrospective cohort study enrolled patients who had undergone pathological examinations for CMV enteritis. They were divided into CMV and non-CMV groups according to immunohistochemistry staining results. The risk factors, clinical presentations, endoscopic features, treatments, outcomes, and prognostic factors of CMV enteritis were then analyzed.
\end{abstract}

Results: Forty-two patients (18 CMV, 24 non-CMV group) were included in the study. Major clinical presentations in the CMV group included gastrointestinal bleeding (72.2\%), abdominal pain (55.6\%), and fever (33.3\%); ulcers (72.2\%) were the most common endoscopic findings. In-hospital and overall mortality rates were $27.8 \%$ and 38.9\%, respectively; and longer hospital stays and higher overall mortality rates were observed. Radiotherapy and C-reactive protein levels were prognostic factors for in-hospital mortality. The risk factors for CMV enteritis included immunocompromised status $(p=0.013)$, steroid use $(p=0.014)$, shock $(p=0.031)$, concurrent pneumonia $(p=0.01)$, antibiotic exposure $(p<0.001)$, radiotherapy $(p=0.027)$, chronic kidney disease $(p=0.041)$, and CMV colitis $(p=0.031)$.

Conclusions: Physicians should pay attention to the characteristics of CMV enteritis in high-risk patients to make an early diagnosis and potentially improve the clinical outcome.

Keywords: Cytomegalovirus enteritis, Endoscopy, Risk factor, Prognostic factor, Immunohistochemistry staining

\section{Background}

Cytomegalovirus (CMV) is a double-stranded DNA virus that has a global seroprevalence rate of up to $83 \%$ in the general population [1]. It causes not only subclinical infections but also serious tissue invasive diseases. The gastrointestinal (GI) tract is a common location for CMV infection, both in immunocompromised as well as immunocompetent patients; it has variable clinical

\footnotetext{
*Correspondence: puohsien@gmail.com

2 Department of Gastroenterology and Hepatology, Chang Gung Memorial Hospital, Linkou Branch, Taoyuan, Taiwan

Full list of author information is available at the end of the article
}

presentations and a high mortality rate [2-5]. Research of small intestinal CMV disease is relatively rare compared to other GI tract CMV diseases possibly due to difficult tissue sampling.

Prior studies on CMV enteritis were limited to case reports or small case series that established the diagnosis by serological tests and did not analyze small intestinal CMV disease separately [6-11]. For example, Kusne et al. reported 21 episodes of CMV enteritis after intestinal transplantation, but the diagnosis was established by histopathology or virology methods [12]. A literature review of CMV GI diseases in immunocompetent hosts original author(s) and the source, provide a link to the Creative Commons licence, and indicate if changes were made. The images or other third party material in this article are included in the article's Creative Commons licence, unless indicated otherwise in a credit line to the material. If material is not included in the article's Creative Commons licence and your intended use is not permitted by statutory regulation or exceeds the permitted use, you will need to obtain permission directly from the copyright holder. To view a copy of this licence, visit http://creativecommons.org/licenses/by/4.0/. The Creative Commons Public Domain Dedication waiver (http://creativeco mmons.org/publicdomain/zero/1.0/) applies to the data made available in this article, unless otherwise stated in a credit line to the data. 
has listed seven cases with small intestine involvement where the diagnosis was confirmed by either biopsy or serological tests, with no detailed clinical or endoscopic information provided [7]. Peter et al. reported the endoscopic findings of cytomegalovirus infection of the upper gastrointestinal tract in solid organ transplant recipients, but the clinical presentations and endoscopic features were statistically analyzed along with CMV gastritis [13]. The latest single-center study reported the characteristics, clinical manifestations, prognosis, and factors associated with gastrointestinal cytomegalovirus infection in immunocompetent patients, but did not analyze CMV small intestinal disease separately [11]. To the best of our knowledge, no study has comprehensively analyzed small intestine CMV disease, including the risk factors, clinical manifestations, endoscopic features, diagnosis, treatments, outcomes, and prognostic factors.

\section{Methods}

\section{Declarations}

The study protocol was approved by the Institutional Review Board of the Chang Gung Medical Foundation on May 14, 2020 for the period of May 01, 2020-May 01, 2021. The Institutional Review Board did not require signed informed consent from individual patients to review medical records from the electronic medical record system, in retrospective studies. The study protocol conformed to the ethical guidelines of the 1975 Declaration of Helsinki, as reflected in a prior approval by the institution's human research committee.

\section{Patients}

In this retrospective cohort study, we enrolled patients from the pathology database at the Linkou Chang Gung Memorial Hospital, who underwent small intestine CMV immunohistochemistry (IHC) staining between January, 2000 and February, 2021. CMV enteritis was diagnosed based on positive CMV IHC staining of the enteric tissue, with or without viral inclusion bodies, using hematoxylin and eosin staining (Fig. 1). CMV IHC was performed using monoclonal antibodies directed against the CMV pp65 antigen (Novocastra ${ }^{\mathrm{TM}}$ lyophilized mouse monoclonal antibody; Leica Microsystems, Wetzlar, Germany). The patients were further divided into two groups (CMV and non-CMV) according to the pathological results.

\section{Data collection}

The medical records of eligible patients were reviewed for data on age, sex, patient source (inpatient, outpatient), admission date, diagnostic date, recurrence date, death or last follow-up, presence of critical condition within 1 week before diagnosis (shock, respiratory distress with ventilator usage), underlying disease, medication history, major clinical presentation, endoscopic findings (lesion characteristics, location, number, and concomitant mucosal findings), histopathology results (presence of malignancy or findings indicating other etiology of enteritis), treatment and therapeutic duration, complications, outcomes (in-hospital mortality rate and overall mortality rate), total white blood cell count, absolute neutrophil count, absolute lymphocyte count, platelet count, hemoglobin $(\mathrm{Hb})$, creatinine $(\mathrm{Cr})$, aspartate aminotransferase,

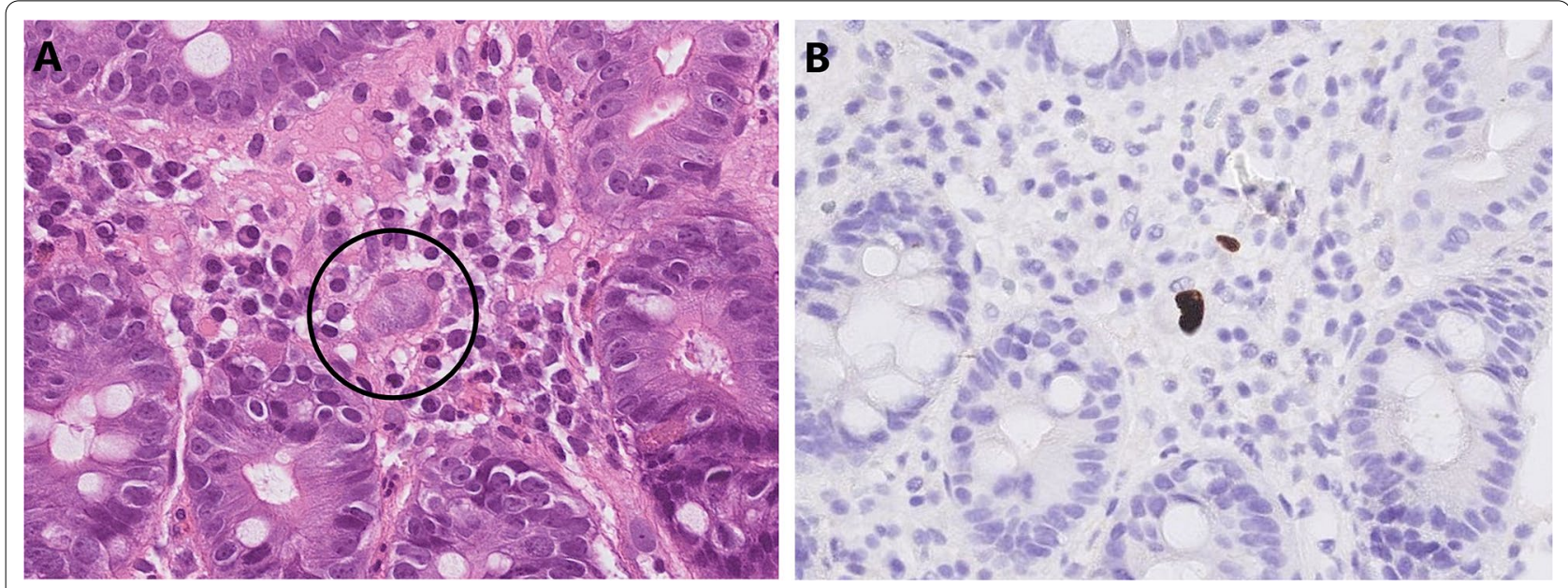

Fig. 1 Pathological presentations of CMV enteritis. CMV enteritis was diagnosed using CMV inclusion bodies and IHC staining of the enteric tissue. A H\&E staining ( $\times 40$ objective) showing typical intranuclear (owl's eye) and intracytoplasmic (eosinophilic punctiform) CMV inclusions within the circles. B $\mathrm{HC}$ staining ( $\times 20$ objective) was performed with 1:200 diluted Novocastra ${ }^{\mathrm{TM}}$ lyophilized mouse monoclonal antibody against CMV pp65 antigen and showed strong focal CMV immunoreactivity with brownish areas. CMV cytomegalovirus, H\&E hematoxylin and eosin, IHC immunohistochemistry 
alanine aminotransferase, bilirubin, albumin, C-reactive protein (CRP) levels, CMV pp65 antigenemia, CMV DNA (226 bp segment on glycoprotein B gene, LightMix ${ }^{\circledR}$ Kit human cytomegalovirus; TIB Molbiol, Berlin, Germany), and CMV serology.

\section{Definition of immune status}

Patients were defined as "immunocompromised" if they were documented to have primary immunodeficiency, human immunodeficiency virus infection, use of chemotherapeutic agents within 6 months, use of immunosuppressants [including corticosteroids (oral or intravenous administration, $\geqq 20 \mathrm{mg} /$ day of prednisolone or any equivalent for $>2$ weeks)], or were recipients of solid organ or bone marrow transplantation $[3,11]$.

\section{Statistical analyses}

Numerical data are presented as mean \pm standard deviation or median (interquartile range), while categorical data are expressed as absolute numbers and percentages. Independent $\mathrm{t}$-tests and Mann-Whitney $\mathrm{U}$ tests were used to compare continuous variables, while $x^{2}$ and Fisher's exact tests were used for categorical variables. Logistic regression models were used to identify the independent risk factors for in-hospital mortality. Statistical significance was set at $\mathrm{p}<0.05$. The results are presented as odds ratios (ORs), 95\% confidence intervals (CIs), and p-values. Survival outcomes were evaluated using Kaplan-Meier survival curve analysis and log-rank test. All statistical calculations were performed using the SPSS statistical software, version 21.0 (Armonk, NY: IBM Corp.).

\section{Results}

\section{Demographics of CMV enteritis}

Forty-two patients were enrolled, including 18 in the CMV group and the rest in the non-CMV group. In the CMV group, 15 (83.3\%) were in-hospital patients, and four of them needed intensive care. The mean age was $49.8 \pm 19.9$ years, and males were predominant (66.7\%). Most patients with CMV enteritis (77.8\%) were immunocompromised. The major comorbidities were chronic kidney disease, malignancy, and hypertension. The types of malignancies were diverse but mostly involved solid organs [lung, brain, bladder, prostate, ileum (adenocarcinoma), liver (cholangiocarcinoma), and skin]. One patient with ileal adenocarcinoma was diagnosed and treated before the development of CMV enteritis. Three patients had undergone renal transplantation (without antiviral prophylaxis), and three had autoimmune diseases (systemic lupus erythematosus, autoimmune hepatitis, and Behcet's disease). CMV colitis and CMV gastritis (concurrent diagnosis or past history) were noted in six and one patient, respectively. More patients with Crohn's disease were enrolled in the non-CMV group because physicians always took biopsy specimens from terminal ileal ulcers to confirm the diagnosis of Crohn's disease and concurrent CMV infection. Other details and differences between the two groups are presented in Table 1.

\section{Clinical manifestations of $\mathrm{CMV}$ enteritis}

The major symptoms of CMV enteritis include GI bleeding (more hematochezia than melena), abdominal pain, diarrhea, and fever. Only one patient presented with intestinal perforation at the time of diagnosis: this 4-yearold boy with newly diagnosed Crohn's disease developed ileal perforation after receiving around 2 weeks of corticosteroids.

\section{Diagnosis of CMV enteritis}

Anemia, hypoalbuminemia, and elevated CRP levels were noted in patients with CMV enteritis. As for virology tests, all the patients were positive for CMV-IgG, however, only around one-third were positive for either CMV-IgM, viremia, or CMV pp65 antigenemia.

Regarding the methods of tissue sampling, colonoscopy (in 11 patients, including 1 with sigmoidoscopy) and esophagogastroduodenoscopy (in 3 patients) were the two major modalities, while per-anus double balloon enteroscopy was performed in 2 patients. Before the index endoscopy, which confirmed the diagnosis, eight patients (44.4\%) had undergone more than one endoscopic examination, indicating difficulty in confirmation of the diagnosis, resulting in the need for repeated approaches. Endoscopically, ulcers were the leading presentation, followed by polypoid lesions and inflammation (in the absence of ulcer or mass-like change) (Fig. 2). Regarding location, the ileum was the most frequently involved segment. Histopathological samples from five patients with CMV enteritis did not show typical CMV viral inclusion bodies, but atypical lymphocytes or prominent germinal centers with inflammatory infiltrates.

In this study, the mean time to diagnosis [from admission (or first visit to the outpatient clinic) to diagnosis of CMV enteritis] was $18.9 \pm 15.8$ days, ranging from 2 to 63 days.

\section{Treatments and outcomes of CMV enteritis}

In total, 12 patients $(66.7 \%)$ received antiviral therapy (intravenous ganciclovir or oral valganciclovir), with a variable duration range (intravenous: 6-23 days, oral: 7-194 days). Three patients underwent surgeries for CMV enteritis to bypass the stricture or repair the intestinal perforation. The mean duration of admission was $35.8 \pm 15.6$ days, and the median follow-up duration was 
Table 1 Baseline characteristics of the patients with or without CMV enteritis

\begin{tabular}{|c|c|c|c|c|}
\hline Characteristics & Overall $(n=42)$ & CMV enteritis $(n=18)$ & Non-CMV enteritis $(n=24)$ & $p$-value \\
\hline Age, years & $49.7 \pm 21.5$ & $49.8 \pm 19.9$ & $49.5 \pm 23$ & 0.809 \\
\hline $\operatorname{Sex}(M / F)$ & $24(57.1 \%) / 18(42.9 \%)$ & $12(66.7 \%) / 6(33.3 \%)$ & $12(50 \%) / 12(50 \%)$ & 0.353 \\
\hline \multicolumn{5}{|l|}{ General conditions } \\
\hline Shock & $7(16.7 \%)$ & $6(33.3 \%)$ & $1(4.2 \%)$ & $0.031^{*}$ \\
\hline Pneumonia & $5(11.9 \%)$ & $5(27.8 \%)$ & $0(0 \%)$ & $0.01^{*}$ \\
\hline Intubation & $5(11.9 \%)$ & $4(22.2 \%)$ & $1(4.2 \%)$ & 0.146 \\
\hline ICU required & $6(14.3 \%)$ & $4(22.2 \%)$ & $2(8.3 \%)$ & 0.375 \\
\hline \multicolumn{5}{|l|}{ Underlying diseases } \\
\hline Immunocompromised & $23(54.8 \%)$ & $14(77.8 \%)$ & $9(37.5 \%)$ & $0.013^{*}$ \\
\hline Diabetes mellitus & $4(9.5 \%)$ & $1(5.6 \%)$ & $3(12.5 \%)$ & 0.623 \\
\hline Hypertension & $12(28.6 \%)$ & $6(33.3 \%)$ & $6(25 \%)$ & 0.732 \\
\hline Autoimmune disease & $3(7.1 \%)$ & $3(16.7 \%)$ & $0(0 \%)$ & 0.071 \\
\hline Coronary artery disease & $3(7.1 \%)$ & $3(16.7 \%)$ & $0(0 \%)$ & 0.071 \\
\hline COPD & $1(2.4 \%)$ & $1(5.6 \%)$ & $0(0 \%)$ & 0.429 \\
\hline \multicolumn{5}{|l|}{ Renal disease } \\
\hline AKI & $3(7.1 \%)$ & $3(16.7 \%)$ & $0(0 \%)$ & 0.071 \\
\hline CKD & $13(31 \%)$ & $9(50 \%)$ & $4(16.7 \%)$ & $0.041^{*}$ \\
\hline ESRD & $6(14.3 \%)$ & $5(27.8 \%)$ & $1(4.2 \%)$ & 0.068 \\
\hline Crohn's disease & $12(28.6 \%)$ & $2(11.1 \%)$ & $10(41.7 \%)$ & $0.042^{*}$ \\
\hline Ulcerative colitis & $1(2.4 \%)$ & $1(5.6 \%)$ & $0(0 \%)$ & 0.429 \\
\hline HIV infection & $1(2.4 \%)$ & $1(5.6 \%)$ & $0(0 \%)$ & 0.429 \\
\hline Malignancies & $13(31 \%)$ & $8(44.4 \%)$ & $5(20.8 \%)$ & 0.177 \\
\hline Transplantation & $4(9.5 \%)$ & $3(16.7 \%)$ & $1(4.2 \%)$ & 0.297 \\
\hline Antibiotics usage & $19(45.2 \%)$ & $14(77.8 \%)$ & $5(20.8 \%)$ & $<0.001^{*}$ \\
\hline \multicolumn{5}{|c|}{ Immunosuppressive therapies } \\
\hline Steroid & $28.6(57.4 \%)$ & $9(50 \%)$ & $3(12.5 \%)$ & $0.014^{*}$ \\
\hline Chemotherapy & $3(7.1 \%)$ & $2(11.1 \%)$ & $1(4.2 \%)$ & 0.567 \\
\hline Radiotherapy & $4(9.5 \%)$ & $4(22.2 \%)$ & $0(0 \%)$ & $0.027^{*}$ \\
\hline Immunosuppressant & $10(23.8 \%)$ & $6(33.3 \%)$ & $4(16.7 \%)$ & 0.281 \\
\hline \multicolumn{5}{|l|}{ Laboratory data } \\
\hline WBC count $(/ \mu \mathrm{L})$ & 7200 (5400-9500) & 7050 (5050-9750) & $7200(6000-9500)$ & 0.705 \\
\hline Hemoglobin (g/dL) & $10.4(8.1-12.1)$ & $9.2(7.3-10.7)$ & $11.2(9.6-12.4)$ & $0.027^{*}$ \\
\hline Platelet $\left(\times 1000 / \mathrm{mm}^{3}\right)$ & $231(152-294)$ & $182(143.3-275.8)$ & $261(199-294)$ & 0.175 \\
\hline Creatinine (mg/dL) & $1(0.6-2.1)$ & $1.2(0.7-4)$ & $0.8(0.6-1.2)$ & 0.151 \\
\hline $\operatorname{ALT}(I U / L)$ & $16.5(13.3-21)$ & $19(15-23.8)$ & $15.5(10-18.5)$ & 0.05 \\
\hline Albumin (g/dL) & $3.3(2.5-3.6)$ & $2.9(2.4-3.3)$ & $3.6(3.2-4)$ & 0.067 \\
\hline C-reactive protein (mg/dL) & $30(16.7-115.3)$ & $81.3(24.8-153.3)$ & $21.5(6.3-73.8)$ & $0.027^{*}$ \\
\hline \multicolumn{5}{|l|}{ Virology/serology positivity } \\
\hline CMV-IgM & $3 / 14(21.4 \%)$ & $3 / 10(30 \%)$ & 0/4 (0\%) & 0.506 \\
\hline CMV-lgG & $12 / 14(85.7 \%)$ & 10/10 (100\%) & $2 / 4(50 \%)$ & 0.066 \\
\hline CMV pp65 antigenemia & $3 / 12(25 \%)$ & 3/9 (33.3\%) & 0/3 (0\%) & 0.509 \\
\hline CMV PCR & $5 / 9(55.6 \%)$ & $5 / 7(71.4 \%)$ & $0 / 2(0 \%)$ & 0.167 \\
\hline \multicolumn{5}{|l|}{ Clinical presentation } \\
\hline Fever & $7(16.7 \%)$ & $6(33.3 \%)$ & $1(4.2 \%)$ & $0.031^{*}$ \\
\hline Abdominal pain & $22(52.4 \%)$ & $10(55.6 \%)$ & $12(50 \%)$ & 0.764 \\
\hline Vomiting & $9(21.4 \%)$ & $5(27.8 \%)$ & $4(16.7 \%)$ & 0.462 \\
\hline Diarrhea & $12(28.6 \%)$ & $8(44.4 \%)$ & $4(16.7 \%)$ & 0.084 \\
\hline Abdominal fullness & $11(26.2 \%)$ & $5(27.8 \%)$ & $6(25 \%)$ & 1.000 \\
\hline Gl bleeding & $20(47.6 \%)$ & $13(72.2 \%)$ & $7(29.2 \%)$ & $0.012^{*}$ \\
\hline
\end{tabular}


Table 1 (continued)

\begin{tabular}{|c|c|c|c|c|}
\hline Characteristics & Overall $(n=42)$ & CMV enteritis $(n=18)$ & Non-CMV enteritis $(n=24)$ & $p$-value \\
\hline \multicolumn{5}{|l|}{ Endoscopic features } \\
\hline \multicolumn{5}{|l|}{ Main findings } \\
\hline Ulcer & $35(83.3 \%)$ & $13(72.2 \%)$ & $22(91.7 \%)$ & 0.118 \\
\hline Inflammation & $5(11.9 \%)$ & $2(11.1 \%)$ & $3(12.5 \%)$ & 1.000 \\
\hline Polypoid lesion & $10(23.8 \%)$ & $6(33.3 \%)$ & $4(16.7 \%)$ & 0.281 \\
\hline \multicolumn{5}{|l|}{ Location of lesion } \\
\hline Duodenum & $5(11.9 \%)$ & $3(16.7 \%)$ & $2(8.3 \%)$ & 0.636 \\
\hline Jejunum & $5(11.9 \%)$ & $1(5.6 \%)$ & $4(16.7 \%)$ & 0.371 \\
\hline lleum & $32(76.2 \%)$ & $14(77.8 \%)$ & $18(75 \%)$ & 1.000 \\
\hline \multicolumn{5}{|l|}{ Concurrent findings } \\
\hline CMV gastritis & $1(2.4 \%)$ & $1(5.6 \%)$ & $0(0 \%)$ & 0.429 \\
\hline CMV colitis & $7(16.7 \%)$ & $6(33.3 \%)$ & $1(4.2 \%)$ & $0.031^{*}$ \\
\hline \multicolumn{5}{|l|}{ Outcomes } \\
\hline Perforation & $3(7.1 \%)$ & $1(5.6 \%)$ & $2(8.3 \%)$ & 1.000 \\
\hline Overall mortality & $8(19 \%)$ & $7(38.9 \%)$ & $1(4.2 \%)$ & $0.013^{*}$ \\
\hline In-hospital mortality & $6(14.3 \%)$ & $5(27.8 \%)$ & $1(4.2 \%)$ & 0.068 \\
\hline Hospital stay, days & $42(15-64.5)$ & $35(22.5-48.5)$ & $8.5(4-20.5)$ & $0.001^{*}$ \\
\hline
\end{tabular}

AKI acute kidney injury, $A L T$ alanine aminotransferase, $A S T$ aspartate aminotransferase, $C K D$ chronic kidney disease, CMV cytomegalovirus, COPD chronic obstructive pulmonary disease, ESRD end-stage renal disease, $F$ female, $G /$ gastrointestinal, $I C U$ intensive care unit, $I Q R$ interquartile range, $M$ male, $S D$ standard deviation, $W B C$ white blood cell

${ }^{*} \mathrm{P}<0.05$. Age is presented as mean \pm standard deviation. Laboratory data, hospital stay, and follow-up duration are presented as median (IQR). The remaining data were presented as numbers (percentages)

58 (35-209) days. The in-hospital and overall mortality rates were $27.8 \%$ and $38.9 \%$, respectively. No disease recurrence was observed during the follow-up period.

\section{Prognostic factors associated with the in-hospital mortality of CMV enteritis}

History of radiotherapy (OR, 18; 95\% CI 1.194-271.461; $\mathrm{p}=0.037)$ and CRP levels (OR, $1.028 ; 95 \%$ CI $1.001-$ $1.057 ; \mathrm{p}=0.045)$ were the only two independent prognostic factors for in-hospital mortality (Table 2). In the Kaplan-Meier survival curve analysis, patients with CMV enteritis having a history of radiotherapy had significantly poorer survival rates than those without a history of radiotherapy ( $\log$-rank $\mathrm{p}=0.013$ ) (Additional file 1: Figure S1). Antiviral therapy (OR, 2.5; 95\% CI $0.214-29.254 ; \mathrm{p}=0.465)$ did not significantly influence the survival outcome.

\section{Comparison between CMV and non-CMV groups}

More patients in the CMV group had an immunocompromised status, chronic kidney disease, shock, pneumonia, and a history of CMV colitis than those in the non-CMV group. Moreover, a higher proportion of patients in the CMV group had exposure to steroids, antibiotics, and radiotherapy than those in the non-CMV group. Clinically, the CMV group had a higher prevalence of GI bleeding and fever, than the non-CMV group, which contributed to lower $\mathrm{Hb}$ levels and higher CRP levels. With respect to outcomes, patients in the CMV group had significantly longer admission duration and higher overall mortality rate than those in the non-CMV group.

\section{Discussion}

CMV enteritis is a rare but fatal disease that has been under diagnosed. It is not only an opportunistic infection in immunocompromised patients, but also affects immunocompetent individuals [2, 3, 10, 14]. Despite the development of small intestinal endoscopies, such as capsule endoscopy, single-balloon enteroscopy, and doubleballoon enteroscopy, diagnosis of CMV enteritis is still difficult due to critical general condition of the patients, various clinical presentations, and expensive procedure fees. Eight patients (44.4\%) underwent more than one endoscopic examination before the final diagnosis. The time to diagnosis in our series was approximately 3 weeks; however, no similar data were available in the literature for comparison. Nevertheless, these facts imply the importance of repeated endoscopic biopsies in highrisk patients.

In view of histopathological diagnosis of CMV enteritis, a discrepancy between the results of H\&E staining and IHC staining has been reported [15]. The specificity 

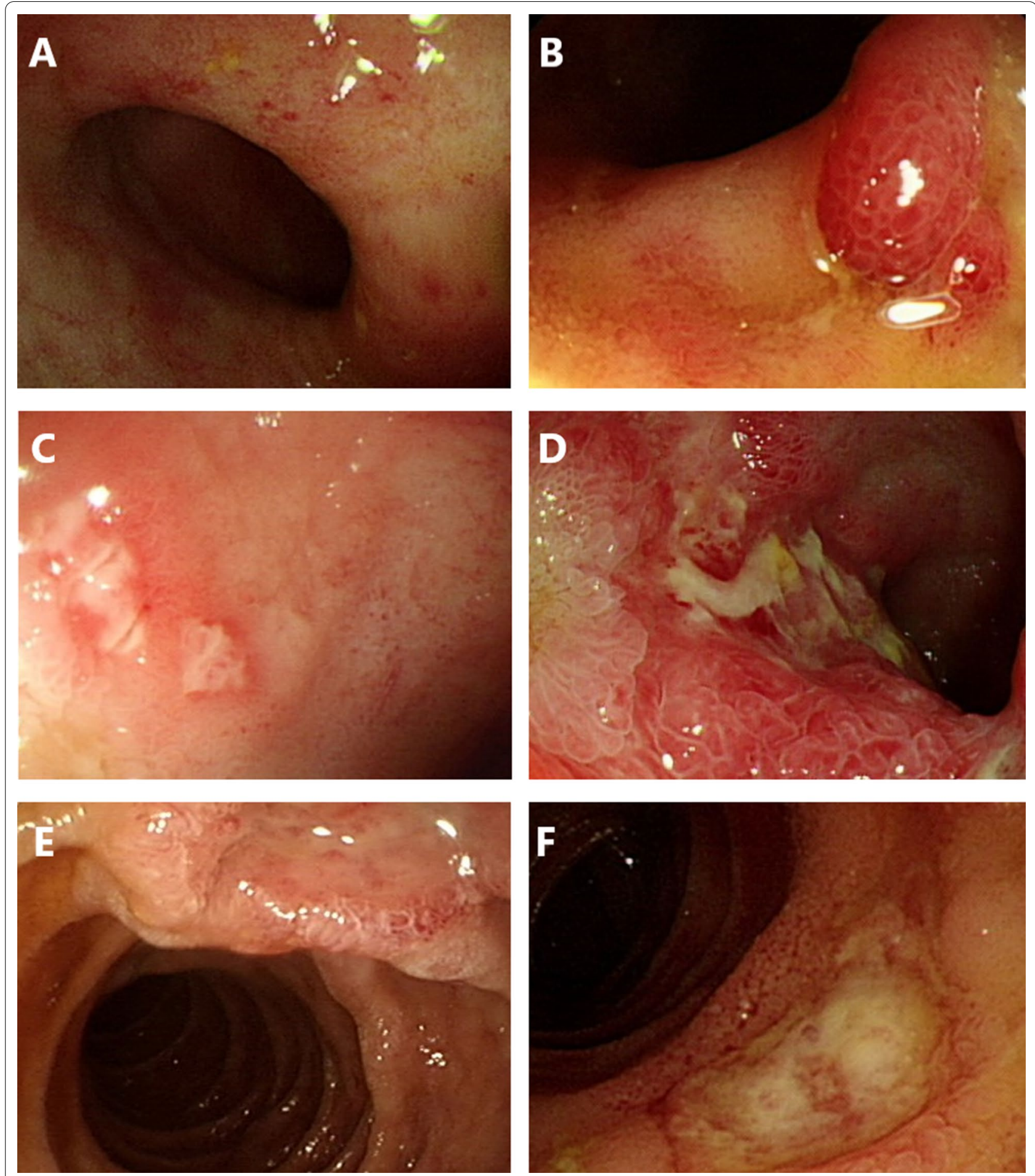

Fig. 2 Endoscopic features of CMV enteritis. A Inflammation. B Polypoid lesion. C-F Variable morphologies of enteric ulcers. CMV cytomegalovirus

and sensitivity of CMV IHC staining has been reported to approach $93 \%$ and $100 \%$ [14], respectively; however, the cytopathic effects are observed in only about $65 \%$ of cells that show positive CMV IHC staining [16]. Therefore, we established positive IHC staining as the diagnosis for CMV enteritis. 
Table 2 Analysis of clinical factors associated with in-hospital mortality

\begin{tabular}{|c|c|c|c|}
\hline \multirow[t]{2}{*}{ Characteristics } & \multicolumn{3}{|c|}{ Univariable analysis } \\
\hline & OR & $95 \% \mathrm{Cl}$ & $p$-value \\
\hline Age & 1.308 & $0.97-1.111$ & 0.285 \\
\hline Sex (male) & 0.667 & $0.078-5.678$ & 0.711 \\
\hline Time to diagnosis & 1.031 & $0.966-1.1$ & 0.365 \\
\hline \multicolumn{4}{|l|}{ General conditions } \\
\hline Shock & 5 & $0.551-45.391$ & 0.153 \\
\hline Pneumonia & 2.222 & $0.245-20.174$ & 0.478 \\
\hline Intubation & 3.667 & $0.354-38.029$ & 0.276 \\
\hline ICU required & 3.667 & $0.354-38.029$ & 0.276 \\
\hline Immunocompromised & $897,486,006$ & 0 & 0.999 \\
\hline \multicolumn{4}{|l|}{ Underlying diseases } \\
\hline Diabetes mellitus & 0 & 0 & 1 \\
\hline Hypertension & 0.4 & $0.034-4.681$ & 0.465 \\
\hline Autoimmune & 1.375 & $0.096-19.643$ & 0.814 \\
\hline Acute kidney injury & 1.375 & $0.096-19.643$ & 0.814 \\
\hline Chronic kidney disease & 1.75 & $0.215-14.224$ & 0.601 \\
\hline End stage renal disease & 0.563 & $0.047-6.769$ & 0.65 \\
\hline Malignancy & 9 & $0.748-108.31$ & 0.083 \\
\hline Transplantation & 0 & 0 & 0.999 \\
\hline Steroid & 1.75 & $0.215-14.224$ & 0.601 \\
\hline Chemotherapy & 3 & $0.15-59.89$ & 0.472 \\
\hline Radiotherapy & 18 & $1.194-271.461$ & $0.037^{*}$ \\
\hline \multicolumn{4}{|l|}{ Laboratory data } \\
\hline WBC count & 1 & 1 & 0.39 \\
\hline Hemoglobin & 0.76 & $0.496-1.163$ & 0.206 \\
\hline Platelet & 0.994 & $0.983-1.006$ & 0.362 \\
\hline Creatinine & 1.046 & $0.665-1.645$ & 0.845 \\
\hline ALT & 0.966 & $0.885-1.053$ & 0.432 \\
\hline Albumin & 0.202 & $0.017-2.39$ & 0.205 \\
\hline C-reactive protein & 1.028 & $1.001-1.057$ & $0.045^{*}$ \\
\hline \multicolumn{4}{|l|}{ Clinical symptoms } \\
\hline Fever & 1.5 & $0.176-12.775$ & 0.711 \\
\hline Abdominal pain & 4.667 & $0.404-53.95$ & 0.217 \\
\hline Vomiting & 8.25 & $0.795-85.564$ & 0.077 \\
\hline Diarrhea & 0.778 & $0.096-6.322$ & 0.814 \\
\hline Abdominal fullness & 2.222 & $0.245-20.174$ & 0.478 \\
\hline Gl bleeding & 1.778 & $0.148-21.395$ & 0.65 \\
\hline \multicolumn{4}{|l|}{ Endoscopic features } \\
\hline Ulcer & 1.778 & $0.148-21.395$ & 0.65 \\
\hline Inflammation & 3 & $0.15-59.89$ & 0.472 \\
\hline Polypoid lesion & 0 & 0 & 0.999 \\
\hline Duodenum & 1.375 & $0.096-19.643$ & 0.814 \\
\hline Jejunum & $5,250,293,239$ & 0 & 1 \\
\hline Ileum & 0.273 & $0.026-2.829$ & 0.276 \\
\hline Antiviral treatment & 2.5 & $0.214-29.254$ & 0.465 \\
\hline
\end{tabular}

ALT alanine aminotransferase, $C l$ confidence interval, $F$ female, $G l$ gastrointestinal, $I C U$ intensive care unit, $M$ male, $O R$ odds ratio, WBC white blood cell

${ }^{*} \mathrm{P}<0.05$, calculated by logistic regression analysis
Higher CRP levels and history of radiotherapy were the poor prognostic factors for in-hospital mortality. Higher CRP levels represent severe inflammation and infection, leading to a higher in-hospital mortality rate [17]. Radiotherapy can result in acute and chronic bowel damage $[18,19]$, because ionizing radiations cause inflammation, mucosal cell loss, swelling of the endothelial lining of arterioles, ischemia, and neovascularization, all of which contribute to mucosal friability and fibrosis [20]. In this study, three out of four patients with a history of radiotherapy were irradiated in the pelvic and lower spinal regions, which probably jeopardized their bowel integrity, leading to a worse outcome.

In this cohort study, the clinical features and risk factors of CMV enteritis were analyzed by comparing the $\mathrm{CMV}$ and non-CMV groups. In the CMV enteritis group, a significantly higher proportion of patients developed fever and GI bleeding in combination with higher CRP and lower $\mathrm{Hb}$ levels, indicating more severe mucosal injury and inflammation, than that in the non-CMV group. The risk factors for CMV enteritis can be categorized into three categories: predisposing mucosal injury (radiotherapy), comorbidities (CMV colitis), and immunodeficiency (immunocompromised status, shock, concurrent pneumonia, corticosteroid exposure, and chronic kidney disease). As stated earlier, radiotherapy leads to acute and chronic mucosal injury, contributing to the vulnerability and risk of superimposed infection. Second, CMV colitis is a risk factor for CMV enteritis because they share similar risk factors; this association was first mentioned in our study. Third, in addition to immunocompromised status, critical illness (shock, concurrent pneumonia), corticosteroid exposure, and chronic kidney disease lead to immunodeficiency [21, 22]. In addition, antibiotics are commonly prescribed for critical conditions, such as severe pneumonia and shock. Therefore, CMV enteritis should be considered when high-risk patients suffer from fever and GI bleeding.

The limitations of this study include the small number of cases, retrospective design, and single-center-based resources. However, it may not be feasible to conduct a prospective study for this rare yet frequently fatal disease.

\section{Conclusion}

This is the first cohort study to demonstrate the comprehensive features of CMV enteritis (small intestine), treatments, outcomes, risk factors, and prognostic factors with scrupulous diagnostic criteria. Radiotherapy, CMV colitis, and immunodeficiency are risk factors for CMV enteritis. Fever and GI bleeding are the most common symptoms. Meanwhile, radiotherapy and higher CRP levels are poor prognostic factors for inhospital mortality. Physicians should be aware of these 
characteristics, which could enable them to make an early diagnosis and potentially improve the clinical outcome.

\section{Supplementary Information}

The online version contains supplementary material available at https://doi. org/10.1186/s13099-021-00450-4.

Additional file 1: Figure S1. Kaplan-Meier survival curve analysis of CMV enteritis patients with/without radiotherapy. Patients with CMV enteritis who received radiotherapy (black line) had a significantly worse survival rate than those who did not receive radiotherapy (gray line) (log-rank $\mathrm{p}=0.013)$. CMV, cytomegalovirus.

\section{Acknowledgements}

The authors are grateful to Yu-Bin Pan (Biostatistical Section, Clinical Trial Center, Chang Gung Memorial Hospital "MOHW110-TDU-B-212-124005") for the statistical support.

\section{Authors' contributions}

Planning and conducting the study: PHL and CTC. Collecting data and statistical analysis: PJY and PHL. Literature review: CJK, JTH, WPL, THC. Data interpretation: PJY, MYS, CMC. Drafting the manuscript: PJY, MWL. Pathological consultation and review: RCW. Critical revision of manuscript: PHL, MWL. All authors read and approved the final manuscript.

\section{Funding}

This study was not funded by any grant or other financial sponsor. The authors have no financial arrangement with a company whose product is discussed in this manuscript.

\section{Availability of data and materials}

The datasets used and/or analysed during the current study are available from the corresponding author on reasonable request.

\section{Declarations}

\section{Ethics approval and consent to participate}

The study protocol was approved by the Institutional Review Board of the Chang Gung Medical Foundation on May 14, 2020 (Approval Document No 202000808BO "Clinical presentations and outcomes of cytomegalovirus infection") for the period of May 01, 2020-May 01, 2021.

\section{Consent for publication}

Not applicable.

\section{Competing interests}

The authors declare that they have no competing interests.

\section{Author details}

'Department of Pediatric Gastroenterology, Chang Gung Memorial Hospital, Linkou Branch, Taoyuan, Taiwan. ${ }^{2}$ Department of Gastroenterology and Hepatology, Chang Gung Memorial Hospital, Linkou Branch, Taoyuan, Taiwan.

${ }^{3}$ Taiwan Association of the Study of Small Intestinal Disease, Taoyuan, Taiwan. ${ }^{4}$ Liver Research Center, Chang Gung Memorial Hospital, Linkou Branch, Taoyuan, Taiwan. ${ }^{5}$ Department of Pathology, Chang Gung Memorial Hospital, Linkou Branch, Taoyuan, Taiwan. ${ }^{6}$ Department of Medical Imaging and Interventions, Chang Gung Memorial Hospital, Linkou Branch, Taoyuan, Taiwan. ${ }^{7}$ Department of General Surgery, Chang Gung Memorial Hospital, Linkou Branch, Taoyuan, Taiwan. ${ }^{8}$ Department of Gastroenterology and Hepatology, New Taipei City Municipal Tucheng Hospital (Chang Gung Memorial Hospital, Tucheng Branch), New Taipei City, Taiwan.

Received: 1 May 2021 Accepted: 12 August 2021 Published online: 18 August 2021

\section{References}

1. Zuhair M, Smit GSA, Wallis G, Jabbar F, Smith C, Devleesschauwer B, et al. Estimation of the worldwide seroprevalence of cytomegalovirus: a systematic review and meta-analysis. Rev Med Virol. 2019;29(3):e2034.

2. Yeh PJ, Chiu CT, Lai MW, Wu RC, Kuo CJ, Hsu JT, et al. Cytomegalovirus gastritis: clinicopathological profile. Dig Liver Dis. 2021;53(6):722-8.

3. Le PH, Lin WR, Kuo CJ, Wu RC, Hsu JT, Su MY, et al. Clinical characteristics of cytomegalovirus colitis: a 15-year experience from a tertiary reference center. Ther Clin Risk Manag. 2017;13:1585-93.

4. Le PH, Kuo CJ, Wu RC, Hsu JT, Su MY, Lin CJ, et al. Pancolitis associated with higher mortality risk of cytomegalovirus colitis in patients without inflammatory bowel disease. Ther Clin Risk Manag. 2018;14:1445-51.

5. Wang HW, Kuo CJ, Lin WR, Hsu CM, Ho YP, Lin CJ, et al. The clinical characteristics and manifestations of cytomegalovirus esophagitis. Dis Esophagus. 2016;29(4):392-9.

6. van Burik JA, Lawatsch EJ, DeFor TE, Weisdorf DJ. Cytomegalovirus enteritis among hematopoietic stem cell transplant recipients. Biol Blood Marrow Transplant. 2001;7(12):674-9.

7. Cha JM, Lee JI, Choe JW, Joo KR, Jung SW, Shin HP, et al. Cytomegalovirus enteritis causing ileal perforation in an elderly immunocompetent individual. Yonsei Med J. 2010;51(2):279-83.

8. Reggiani Bonetti L, Losi L, Di Gregorio C, Bertani A, Merighi A, Bettelli S, et al. Cytomegalovirus infection of the upper gastrointestinal tract: a clinical and pathological study of 30 cases. Scand J Gastroenterol. 2011;46(10):1228-35.

9. Ozaki T, Yamashita H, Kaneko S, Yorifuji H, Takahashi H, Ueda Y, et al. Cytomegalovirus disease of the upper gastrointestinal tract in patients with rheumatic diseases: a case series and literature review. Clin Rheumatol. 2013;32(11):1683-90.

10. Karigane D, Takaya S, Seki Y, Mastumoto Y, Onose A, Kosakai A, et al. Cytomegalovirus enteritis in immunocompetent subjects: a case report and review of the literature. J Infect Chemother. 2014;20(5):325-9.

11. Chaemsupaphan T, Limsrivilai J, Thongdee C, Sudcharoen A, Pongpaibul A, Pausawasdi N, et al. Patient characteristics, clinical manifestations, prognosis, and factors associated with gastrointestinal cytomegalovirus infection in immunocompetent patients. BMC Gastroenterol. 2020;20(1):22.

12. Kusne S, Mañez R, Frye BL, St George K, Abu-Elmagd K, Tabasco-Menguillon $J$, et al. Use of DNA amplification for diagnosis of cytomegalovirus enteritis after intestinal transplantation. Gastroenterology. 1997;112(4):1121-8.

13. Peter A, Telkes G, Varga M, Sarvary E, Kovalszky I. Endoscopic diagnosis of cytomegalovirus infection of upper gastrointestinal tract in solid organ transplant recipients: Hungarian single-center experience. Clin Transplant. 2004;18(5):580-4.

14. You DM, Johnson MD. Cytomegalovirus infection and the gastrointestinal tract. Curr Gastroenterol Rep. 2012;14(4):334-42.

15. Juric-Sekhar G, Upton MP, Swanson PE, Westerhoff M. Cytomegalovirus (CMV) in gastrointestinal mucosal biopsies: should a pathologist perform CMV immunohistochemistry if the clinician requests it? Hum Pathol. 2017;60:11-5.

16. Fakhreddine AY, Frenette CT, Konijeti GG. A practical review of cytomegalovirus in gastroenterology and hepatology. Gastroenterol Res Pract. 2019;2019:6156581.

17. Sproston NR, Ashworth JJ. Role of C-reactive protein at sites of inflammation and infection. Front Immunol. 2018:9:754.

18. Andreyev J. Gastrointestinal complications of pelvic radiotherapy: are they of any importance? Gut. 2005;54(8):1051-4.

19. Andreyev J. Gastrointestinal symptoms after pelvic radiotherapy: a new understanding to improve management of symptomatic patients. Lancet Oncol. 2007:8(11):1007-17.

20. Stacey R, Green JT. Radiation-induced small bowel disease: latest developments and clinical guidance. Ther Adv Chronic Dis. 2014;5(1):15-29.

21. Kato S, Chmielewski M, Honda H, Pecoits-Filho R, Matsuo S, Yuzawa Y, et al. Aspects of immune dysfunction in end-stage renal disease. Clin J Am Soc Nephrol. 2008;3(5):1526-33.

22. Hotchkiss RS, Monneret G, Payen D. Immunosuppression in sepsis: a novel understanding of the disorder and a new therapeutic approach. Lancet Infect Dis. 2013;13(3):260-8.

\section{Publisher's Note}

Springer Nature remains neutral with regard to jurisdictional claims in published maps and institutional affiliations. 